\title{
Tabagismo em amostra de adolescentes escolares de Salvador-Bahia*
}

\section{Smoking among school adolescents in Salvador (BA)}

\author{
Adelmo Souza Machado Neto ${ }^{1}$ (te sbpt), Álvaro A. Cruz (te sbpt)
}

Introdução: O hábito de fumar em geral se inicia na adolescência. No Brasil, as estimativas da freqüência deste hábito entre adolescentes variam de $1 \%$ até $35 \%$.

O bjetivo: Estimar a prevalência do tabagismo entre os adolescentes da oitava série do ensino fundamental à terceira série do curso médio, em escolas de Salvador - Bahia, Brasil.

Método: Feito um estudo do tipo corte transversal de caráter exploratório. Foram aplicados 3.500 questionários a alunos matriculados entre a $8^{a}$ série do ensino fundamental e a 3 a série do ensino médio, em cinco escolas da região metropolitana de Salvador (BA). A nálise estatística: medidas descritivas e de associação (razão de prevalência) e o teste t de Student e o do quiquadrado.

Resultados: A prevalência do tabagismo entre adolescentes de Salvador (BA) foi de 9,6\%, considerando-se os 3.180 questionários válidos, sendo maior no sexo masculino (14\%) que no feminino (6\%). À medida que aumentava a idade, elevava-se essa prevalência. A média de idade do início do tabagismo foi de $14 \pm 2$ anos. Dentre adolescentes, $46 \%$ experimentaram 0 cigarro e $20 \%$ destes continuaram fumando. Entre filhos de fumantes a freqüência foi maior. 0 número médio de cigarros consumidos por dia pelos adolescentes que fumavam diariamente ( $n=132$ ) foi de $7 \pm 6$, sendo maior no sexo masculino.

Conclusão: A prevalência do tabagismo em uma amostra selecionada de adolescentes escolares de Salvador (BA) foi de $9,6 \%$, sendo maior entre os indivíduos do sexo masculino. A experimentação e a influência dos pais foram associadas ao tabagismo nos adolescentes. (J Pneumol 2003;29(5):264-72)

Descritores - Tabagismo/ epidemiologia. A dolescente. Estudantes. Tabaco.
Background: Most tobacco users become addicted during adolescence. In Brazil, smoking prevalence among teenagers varies from $1 \%$ to $35 \%$.

Objective: To estimate the prevalence of smoking among teenagers, aged from 13 to 20, in fundamental and high school in Salvador, Bahia, B razil.

Method: Cross-sectional exploratory study. Thirty five hundred questionnaires were applied to students at fundamental and high school in five schools at the metropolitan region of Salvador (BA). Statistical analysis: descriptive and associative measurements (Prevalence Rate), Student's t and qui-square tests.

Results: Smoking prevalence among teenagers in Salvador (BA) was $9.6 \%$, considering 3,180 valid questionnaires. The frequency was higher in males (14\%) than in females $(6 \%)$. The prevalence increased with age. The mean age \pm SD for tobacco initiation was $14 \pm 2$ years. Among the teenagers, $46 \%$ tried cigarettes, and $20 \%$ became addicted. The frequency was higher among teenagers whose parents were smokers. The mean \pm SD number of daily cigarettes smoked by adolescent tobacco users ( $n=132$ ) was $7.4( \pm 6.4$ ) units per day, with a higher frequency among boys.

Conclusion: Smoking prevalence in a selected set of adolescent students in Salvador (BA) was $9.6 \%$, and it was higher among males. Parental smoking and cigarette experimentation were the major factor found to be associated to tobacco addiction among teenagers.

Key words - Smoking/ epidemiology. A dolescents. Students. Tobacco.

Título de especialista pela Sociedade Brasileira de Pneumologia e Tisiologia.

Endereço para correspondência - Dr. Adelmo Souza Machado Neto, Centro de Enfermidades Respiratórias - Setor Pneumologia - HUPES, Rua Augusto Viana, S/N, 3 andar - 40110-160 - Salvador, BA Brasil. Tel.: (71) 237-6679; fax: (71) 237-6679/247-1585; e-mail: machadon@ufba.br / alvaro@compos.com.br

Recebido para publicação em 4/2/03. Aprovado, após revisão, em 2/6/03. 


\section{INTRODUÇÃO}

O estudo do tabagismo entre adolescentes torna-se importante, pois é na adolescência que ocorre a iniciação ao tabagismo. Há especial vulto quando se considera que ele tem potencial de causar doenças e incapacitações precocemente. ${ }^{(1,2)}$ Doenças que ocorrem freqüentemente entre indivíduos adultos e idosos podem ocorrer também entre jovens. Nesse sentido, dados do Third National Health and Nutrition Examination Survey, nos Estados Unidos, sobre a prevalência de alterações funcionais respiratórias obstrutivas relacionadas ao tabaco, mostraram na faixa etária de 17 a 24 anos freqüência entre 10 e $15 \%$. (3) Além disso, os adolescente fumantes são acometidos com maior freqüência por sintomas e infecções respiratórias, ${ }^{(4,5)}$ por comprometimento da saúde bucal, ${ }^{(6,7)}$ pela redução da capacidade física(8) e pela dependência da nicotina. ${ }^{(9)}$

A pesar do reconhecimento dos malefícios do hábito de consumo do tabaco, este vem mantendo-se constante entre os adolescentes, existindo tendência à diminuição somente entre os adultos. ${ }^{(10)}$ Dados epidemiológicos dos Estados Unidos (1965-1995) revelaram que 15\% a 49\% da população jovem faziam uso do cigarro ${ }^{(10)}$ e que $90 \%$ dos tabagistas adultos iniciaram o hábito em idade igual ou inferior a 18 anos. (2) Nos EUA, a cada dia, três mil crianças tornam-se fumantes ${ }^{(11)}$ e desde 1991 esse hábito vem sendo mantido entre $22 \%$ e $27 \%$ dos adultos jovens, mesmo considerando a redução das propagandas do produto e 0 incremento das campanhas preventivas. ${ }^{(12)}$ J á na União Européia (2000), a prevalência do tabagismo entre adolescentes foi mais freqüente no sexo masculino, sendo que, na França, atingiu a marca de $35 \%{ }^{(13)} \mathrm{Na}$ A mérica Latina, publicações têm revelado números variados: no Chile (1988, 1997 e 1999) entre 10 e 64\%, sendo a média de idade de início de 13,6 anos;:(14-16) na Costa Rica (1987), verificou-se $28,4 \%$ de prevalência nessa faixa etária;(17) na llha de Páscoa (1994), a taxa foi de $50 \%$. ${ }^{(18)}$

No Brasil, a prevalência do hábito entre adolescentes tem variado de $1 \%$ a $37,7 \% .^{(19-22)}$ Dados do Ministério da Saúde (1989) mostraram que no Brasil havia 30,6 miIhões de fumantes, correspondendo a $24,6 \%$ da população acima dos cinco anos de idade, sendo 18 milhões de homens e 12,6 milhões de mulheres. ${ }^{(23)}$ No Brasil (2001), a prevalência do tabagismo entre adolescentes na faixa etária entre 12 e 17 anos foi de 15,7\%, sendo $16,2 \%$ no sexo masculino e $15,2 \%$ no feminino.(20) 0 Ministério da Saúde mostrou que, entre os anos de 1987 e 1997, em 10 capitais brasileiras consideradas, houve aumento da experimentação do cigarro em todas elas. ${ }^{(23)} \mathrm{O}$ utro estudo no Brasil mostrou que $53 \%$ das pessoas acima de 35 anos de idade já haviam experimentado o tabaco. ${ }^{(20)}$ Es-
Siglas e abreviaturas utilizadas neste trabalho

DP - Desvio padrão

IC $95 \%$ - Intervalo de confiança de $95 \%$

RP - Razão de prevalência

pecificamente em Salvador (BA) (1987), foi encontrada prevalência de $1 \%$ nas escolas públicas e particulares, sendo este o último estudo sobre este tema entre escolares na Bahia. ${ }^{(19)}$

Baseado nesses dados epidemiológicos, este estudo visou estimar a prevalência do tabagismo entre os adolescentes da 8a série do ensino fundamental à 3 a série do curso médio, em escolas de Salvador (Bahia-Brasil). Também objetivou identificar a proporção de adolescentes que experimentaram o cigarro e com que idade o fizeram, pois este é o passo principal para a dependência do tabaco entre adolescentes. ${ }^{(24)}$ Buscou-se neste estudo observar se havia associação do tabagismo dos pais com o tabagismo dos filhos, considerando que o uso do cigarro pelos pais pode aumentar as probabilidades dos adolescentes de iniciar o hábito. ${ }^{(24,25)}$

\section{MÉTOdo}

Selecionaram-se para o estudo 3.500 alunos matriculados em cinco escolas da região metropolitana de Salvador (BA) (quatro particulares e uma pública), da 8a série do curso fundamental até a 3 a série do ensino médio, de 13 a 20 anos de idade. 0 instrumento utilizado foi um questionário elaborado pelos autores juntamente com alunos da disciplina de Clínica Medica da Faculdade de Medicina da U niversidade Federal da Bahia. As questões contidas nele têm validade de conteúdo,(26) sendo que este instrumento também abordou objetivos mais amplos (A nexo 1).

As variáveis do questionário selecionadas para este trabalho foram: variáveis categóricas: sexo, hábito de fumar cigarro (fuma com regularidade por pelo menos 30 dias, só em reuniões sociais, só quando bebe ou diariamente), experimentação do cigarro (se o adolescente já fumou para experimentação em qualquer momento da sua vida, considerando seu primeiro contato com o cigarro), tabagismo dos pais (se pai e/ ou mãe fumam), grupo racial baseado na classificação de Krieger et al. (1965) (branco, mulato claro, mulato médio, mulato escuro e negros);(27) variáveis numéricas: idade do adolescente, idade de experimentação do cigarro (quando ocorreu apenas a experimentação do cigarro), idade de início do hábito de fumar (idade que passou a fazer uso regular conforme definição).

O questionário foi aplicado nos referidos alunos, no segundo semestre de 1998, nas salas de aula seleciona- 
das aleatoriamente, em horário letivo, após autorização das diretorias, em presença do professor. Critérios de inclusão: questionários em perfeito estado (sem lacerações, desenhos ou rasuras), os que continham respostas coerentes (aquelas em que os adolescentes responderam às questões dirigidas aos fumantes e que fumavam, e os que responderam as questões que exigiam apenas uma alternativa conforme 0 solicitado), aqueles que foram preenchidos por alunos entre 13 e 20 anos de idade e os que foram respondidos apenas em sala de aula. Critérios de exclusão: aqueles com respostas incoerentes (adolescentes que afirmaram que não fumavam, mas responderam às questões dirigidas aos fumantes e aqueles com questões que exigiam apenas uma alternativa e foi respondida mais de uma), aqueles preenchidos por alunos com idade inferior a 13 anos ou superior a 20 anos, os questionários rasurados, aqueles que foram respondidos fora da sala de aula e os entregues em branco. A amostra escoIhida foi por conveniência dada pela facilidade de acesso. Os dados foram tabulados no programa SPSS $10^{\circledR}$. A análise dos dados foi realizada utilizando-se os programas SPSS $10^{\circledR}$, Epi info versão $6.04^{\circledR} \mathrm{e} \mathrm{PEPI}^{\circledR}$.

O protocolo não foi submetido ao Comitê de Ética em Pesquisa, pois no período de execução do estudo este ainda não estava estruturado no Hospital Universitário Prof. Edgard Santos.

O desenho de estudo consistiu em um estudo de corte transversal de caráter exploratório. Na estimativa da amostra, para o n mínimo necessário para estimativa de uma proporção, consideraram-se o tamanho da população $(91.000),{ }^{(28)}$ a precisão $(5,0 \%)$, a prevalência esperada $(16,0 \%)$, o efeito de desenho $(1,5)$, o nível de confiança $(95 \%)$, resultando de 310 adolescentes; e para comparação entre duas proporções, foram considerados o nível de confiança (95\%), o poder (80\%), a relação expostos/ não expostos $(1: 1,5)$, a prevalência de tabagismo no sexo masculino (12\%), e a prevalência de tabagismo no sexo feminino (6,5\%); este cálculo foi igual a 855; Para a análise estatística, foram feitas medidas descritivas: média e desvio padrão (DP), razão de prevalência (RP), testes estatísticos do qui-quadrado $\left(\chi^{2}\right)$ e teste $t$ de Student (t). 0 nível de significância predeterminado foi de 0,05 e a variabilidade amostral das prevalências e das razões de prevalência foi avaliada utilizando-se cálculo de intervalo de confiança de $95 \%$ (IC 95\%). 0 n do estudo foi de 3.180 questionários válidos, excedendo, portanto o cálculo para o tamanho da amostra, por duas razões: viabilizar a análise de subgrupos e possibilitar a obtenção de $n$ superior ao cálculo amostral.

\section{Resultados}

Dos 3.500 questionários distribuídos, não foram considerados válidos $320(9,1 \%)$, perfazendo um total de 3.180 válidos para o estudo. A prevalência de adolescentes escolares fumantes foi de 9,5\% (303/3.178) (IC 95\% = 8,510,6) (Tabela 1). A prevalência estimada no sexo masculino foi de $14,4 \%(185 / 1.293)$ (IC $95 \%=12,5-16,4)$, e no feminino de $6,2 \%$ (116/1.878) (IC 95\% = 5,4-7,8), sendo a razão de prevalência $=2,33(14,4 / 6,2)$ (IC $95 \%=$ 1,87-2,90) para o sexo masculino (Tabela 1).

A freqüência do tabagismo segundo a idade do adolescente foi de 5,6\% (77/ 1.383) (IC 95\%=4,4-6,9) entre 13 e 15 anos, $11,9 \%$ (171/ 1.438) (IC $95 \%=10,2-13,6)$ entre 16 e 17 anos e $16,4 \%(51 / 311)$ (IC $95 \%=12,4-20,9)$ entre 18 e 20 anos $\left(\chi^{2}=51,4 ; p<0,001\right)$ (Tabela 1$)$.

A prevalência do hábito de fumar, segundo os grupos raciais, foi de $10,9 \%(55 / 504)$ (IC $95 \%=8,3-13,9)$ na raça branca, 9,5\% (133/1.407) (IC 95\% = 8,0-11,1) nos mulatos claros, 9,2\% (76/827) (IC 95\% = 7,3-11,4) entre os mulatos médios, $8,5 \%(18 / 211)$ (IC $95 \%=5,1-13,1)$ entre os mulatos escuros e $8,5 \%$ (11/ 130) (IC $95 \%=4,3$ $14,6)$ entre os negros, sem diferença estatisticamente significante entre elas (Tabela 1 ).

A freqüência de experimentação do cigarro foi de $46 \%$ $(1.455 / 3.170)$ (IC $95 \%=44,2-47,7)$ entre os adolescentes da amostra. Essa freqüência foi de 53,3\% (686/ 1.287$)$ (IC $95 \%=50,5-56,1)$ entre os adolescentes do sexo masculino e $41 \%$ (770/ 1.877) (IC 95\% = 38,8-43,3) entre os do sexo feminino, sendo a experimentação 1,3 vez mais freqüente entre os rapazes $(53,3 / 41,0)$ (IC $95 \%=1,21$ $1,40 ; p<0,001)$. Entre os adolescentes que experimentaram o cigarro, a prevalência do hábito de fumar foi de $20,4 \%$ (297/ 1.455) (IC 95\% = 18,4-22,6) (Tabela 1). A prevalência no sexo masculino dos que experimentaram o cigarro foi de $27 \%(185 / 686)$ (IC $95 \%=23,7-30,5)$ e, no sexo feminino, de $14,6 \%$ (112/769) (IC $95 \%=12,1$ $17,3)$, sendo a $R P=1,85$, mais freqüente no sexo masculino $(27,0 / 14,6)($ IC $95 \%=1,55-2,21)$ (Tabela 1).

A prevalência do tabagismo entre os adolescentes da amostra estudada, segundo a idade de experimentação do cigarro, foi de: $22,3 \%(21 / 94)$ (IC $95 \%=14,47-32,1$ ) quando os adolescentes a fizeram aos 10 anos de idade ou menos, $28,4 \%(126 / 443)$ (IC $95 \%=23,7-30,5)$ quando ocorreu entre 11 e 13 anos de idade, 16,1\% (127/ 787) (IC $95 \%=24,3-32,9)$ quando a realizaram entre 14 e 16 anos e $15,5 \%(11 / 71)$ (IC $95 \%=7,2-26,0)$ quando experimentaram entre 17 e 19 anos $\left(\chi^{2}=127,3\right)$ (Tabela 1). A média de idade $\pm D P$ da experimentação do cigarro $(n=1.392)$ foi de $13,8 \pm 2$ anos entre os adolescentes, com idade mínima de seis anos e máxima de 19 anos. No grupo de fumantes $(n=282)$, a média de idade \pm DP do experimento foi de 13,3 \pm 2 anos e, no de não fumantes $(n=1.110)$, essa média foi de $13,9 \pm 2$ anos. Com relação ao sexo, a média de idade do experimento do cigarro pelos adolescentes do sexo masculino foi de 13,5 \pm 2 anos e por aqueles do sexo feminino foi de $14 \pm 1,9$ anos 
TABELA 1

Prevalência (\%) do tabagismo entre adolescentes escolares em Salvador (BA) segundo o sexo, experimento do cigarro, idade e grupo racial, em 1998

\begin{tabular}{|c|c|c|c|c|}
\hline & \multicolumn{2}{|c|}{ Fuma } & \multirow[b]{2}{*}{$\begin{array}{l}\text { Total* } \\
\text { N (\%) }\end{array}$} & \\
\hline & $\underset{\mathbf{n}(\%)}{\operatorname{Sim}}$ & $\begin{array}{c}\text { Não } \\
\text { n (\%) }\end{array}$ & & \\
\hline Sem informação & - & - & $2(0,1 \%)$ & \\
\hline Prevalência & $303(9,5)$ & $2.875(90,5)$ & 3.178 & \\
\hline \multicolumn{5}{|l|}{ Sexo } \\
\hline Masculino & $186(14,4)$ & $1.107(85,6)$ & 1.293 & $p<0,001$ \\
\hline Feminino & $116(6,2)$ & $1.762(93,8)$ & 1.878 & \\
\hline \multicolumn{5}{|l|}{ Idade (anos) } \\
\hline 13-15 & $77 \quad(5,6)$ & $1.306(94,4)$ & 1.383 & $p<0,001$ \\
\hline $16-17$ & $171(11,9)$ & $1.267(88,1)$ & 1.438 & \\
\hline $18-20$ & $51(16,4)$ & $260(83,6)$ & 311 & \\
\hline \multicolumn{5}{|l|}{ Grupo racial } \\
\hline Brancos & $55(10,9)$ & $449(90,1)$ & 504 & $p=0,799$ \\
\hline Mulatos claros & $133(9,5)$ & $1.274(90,5)$ & 1.407 & \\
\hline Mulatos médios & $76 \quad(9,2)$ & $751(90,8)$ & 827 & \\
\hline Mulatos escuros & $18(8,5)$ & $193(91,5)$ & 211 & \\
\hline Negros & $11(8,5)$ & $119(91,5)$ & 130 & \\
\hline \multicolumn{5}{|l|}{ Experimentação/sexo } \\
\hline Sem informação & - & - & $17(0,5)$ & $p<0,001$ \\
\hline Masculino & $185(27,0)$ & $501(73,0)$ & 686 & \\
\hline Feminino & $112(14,6)$ & $657(85,4)$ & 769 & \\
\hline Total & $297(20,4)$ & $1.158(79,6)$ & 1.455 & \\
\hline \multicolumn{5}{|c|}{ Idade de experimentação (anos) } \\
\hline Sem informação & - & - & $64(4,6)$ & $p<0,001$ \\
\hline$\leq 10$ & $21(22,3)$ & $73(77,7)$ & 94 & \\
\hline $11-13$ & $126(28,4)$ & $317(71,6)$ & 443 & \\
\hline $14-16$ & $127(16,1)$ & $660(83,3)$ & 787 & \\
\hline $17-19$ & $11(15,5)$ & $60(84,5)$ & 71 & \\
\hline
\end{tabular}

(IC $95 \%=-0,67--0,24 ; p<0,001)$. Entre aqueles que experimentaram o cigarro ( $n=1.389$ ), observou-se diferença estatisticamente significante para o sexo dos adolescentes entre as idades 14 e 16 anos ( $p<0,02$ ), assim como entre 17 e 19 anos $(p<0,001)$, conforme visto na Tabela 2.

A freqüência total de pais fumantes foi de $25,3 \%$ (795/ 3.147 ) (IC $95 \%=23,8-26,8$ ), de mães fumantes foi de $21 \%(663 / 3.164)($ IC $95 \%=19,5-20,4)$ e de pai e mãe fumantes, de $8,4 \%(263 / 3.149)$ (IC $95 \%=7,4-9,4)$. A freqüência de pais que faziam uso do cigarro foi de $33,4 \%$ (99/296) (IC $95 \%=7,4-9,4)$ entre os adolescentes fumantes e de $24,4 \%(696 / 2.849)$ (IC $95 \%=22,8-26,1)$ entre os não fumantes. A freqüência de mães fumantes foi de $29,6 \%(89 / 301)$ (IC $95 \%=24,5-35,1)$ entre os fumantes e $20,1 \%(574 / 2.861)$ (IC $95 \%=18,6-21,6)$ entre os não fumantes. A freqüência de pai e mãe fumantes foi de
TABELA 2

D istribuição dos adolescentes que experimentaram o cigarro em Salvador (BA), segundo idade de experimentação e sexo

\begin{tabular}{lcrr}
\hline Idade (anos) & $\begin{array}{c}\text { Masculino } \\
\mathbf{n}(\%)\end{array}$ & $\begin{array}{c}\text { Feminino } \\
\mathbf{n}(\%)\end{array}$ & $\begin{array}{r}\text { Total } \\
\mathbf{n}\end{array}$ \\
\hline$<10^{\text {a }}$ & $51(56,7)$ & $39(43,3)$ & 90 \\
$11-13^{\text {b }}$ & $235(53,2)$ & $207(46,8)$ & 442 \\
$14-16^{c}$ & $340(43,3)$ & $446(56,7)$ & 786 \\
$17-19^{d}$ & $27(38,0)$ & $44(62,0)$ & 71 \\
Total & $653(47,0)$ & $736(53,0)$ & 1.389 \\
\hline * Comparações duas a duas: & a, b: $(p=0,54 ;$ IC $95 \%=0,87-1,30)$ & \\
& a, $c:(p=0,02 ;$ IC $95 \%=1,08-1,60)$ & \\
& a, $d:(p<0,001 ;$ IC $95 \%=6,6-14,9)$ &
\end{tabular}

$13,2 \%(39 / 296)$ (IC $95 \%=9,6-17,7)$ entre os adolescentes fumantes e de 7,9\% (224/2.851) (IC 95\% = 6,9-8,9) entre os não fumantes. 
TABELA 3

Prevalência (\%) do tabagismo entre adolescentes escolares em Salvador (BA) segundo o tabagismo dos pais, em 1998

\begin{tabular}{|c|c|c|c|c|c|c|}
\hline \multirow{3}{*}{$\begin{array}{l}\text { Tabagismo dos pais } \\
\text { Sem informação }\end{array}$} & \multicolumn{2}{|c|}{ Fuma } & \multirow[t]{2}{*}{ Total } & \multirow[t]{2}{*}{ RP* } & \multirow[t]{2}{*}{ IC $95 \% * *$} & \multirow[t]{2}{*}{ Valor de $p$} \\
\hline & Sim & Não & & & & \\
\hline & $7 \quad(2,0 \%)$ & $24 \quad(1,0 \%)$ & $(1,0 \%)$ & - & - & - \\
\hline \multicolumn{7}{|l|}{ Nenhum fuma } \\
\hline N enhum fuma & $152(7,7 \%)$ & $1.813(92,3 \%)$ & $1.965(100,0 \%)$ & 0,62 & $0,50-0,77$ & $<0,001$ \\
\hline Um dos pais fuma & $149(12,5 \%)$ & $1.046(87,5 \%)$ & $1.195(100,0 \%)$ & & & \\
\hline \multicolumn{7}{|l|}{ Apenas o pai } \\
\hline Sim & $99(12,5 \%)$ & $696(87,5 \%)$ & $795(100,0 \%)$ & 1,49 & $1,18-1,87$ & $<0,001$ \\
\hline N ão & $197(8,4 \%)$ & $2.153(91,6 \%)$ & $2.350(100,0 \%)$ & & & \\
\hline \multicolumn{7}{|l|}{ Apenas a mãe } \\
\hline Sim & $89(13,4 \%)$ & $574(86,6 \%)$ & $663(100,0 \%)$ & 1,58 & $1,25-2,0$ & $<0,001$ \\
\hline N ão & $212(8,5 \%)$ & $2.287(91,5 \%)$ & $2.499(100,0 \%)$ & & & \\
\hline \multicolumn{7}{|l|}{ Ambos } \\
\hline Sim & $39(14,8 \%)$ & $224(85,2 \%)$ & $263(100,0 \%)$ & 1,66 & $1,22-2,27$ & $<0,002$ \\
\hline N ão & $257 \quad(8,9 \%)$ & $2.627(91,1 \%)$ & $2.884(100,0 \%)$ & & & \\
\hline
\end{tabular}

* RP = Razão de prevalência; ** IC95\% = Intervalo de confiança $95 \%$

TABELA 4

Prevalência (\%) do hábito de fumar entre adolescentes em Salvador (BA) segundo o tabagismo dos pais e o sexo, em 1998

\begin{tabular}{|c|c|c|c|c|}
\hline \multirow{2}{*}{$\begin{array}{l}\text { Tabagismo dos pais/ } \\
\text { Sexo do adolescente }\end{array}$} & \multicolumn{2}{|c|}{ Fuma } & \multirow[t]{2}{*}{ Total } & \multirow[t]{2}{*}{ Valor de $p$} \\
\hline & Sim & Não & & \\
\hline \multicolumn{5}{|l|}{$\begin{array}{l}\text { Apenas o pai fuma } \\
\text { Masculino }\end{array}$} \\
\hline Sim & $60(18,4 \%)$ & $266(81,6 \%)$ & 326 & $<0,001$ \\
\hline Não & $121(12,7 \%)$ & $831(87,3 \%)$ & 952 & \\
\hline \multicolumn{5}{|l|}{ Feminino } \\
\hline Sim & $39(8,3 \%)$ & $429(91,7 \%)$ & 468 & $<0,022$ \\
\hline Não & $75 \quad(5,4 \%)$ & $1.317(94,6 \%)$ & 1.392 & \\
\hline \multicolumn{5}{|l|}{ Apenas a mãe fuma } \\
\hline Sim & $54(19,9 \%)$ & $218(81,1 \%)$ & 272 & $<0,004$ \\
\hline Não & $131(12,9 \%)$ & $882(87,1 \%)$ & 1.013 & \\
\hline \multicolumn{5}{|l|}{ Feminino } \\
\hline Sim & $35 \quad(9,0 \%)$ & $355(91,0 \%)$ & 390 & $<0,009$ \\
\hline Não & $80 \quad(5,4 \%)$ & $1.400(94,6 \%)$ & 1.480 & \\
\hline \multicolumn{5}{|l|}{ Pai e mãe fumam } \\
\hline \multicolumn{5}{|l|}{ M asculino } \\
\hline Sim & $25(22,1 \%)$ & $88(77,9 \%)$ & 113 & $<0,011$ \\
\hline Não & $156(13,4 \%)$ & $1.009(86,6 \%)$ & 1.165 & \\
\hline \multicolumn{5}{|l|}{ Feminino } \\
\hline Sim & $14 \quad(9,4 \%)$ & $135(90,6 \%)$ & 149 & \\
\hline Não & $100 \quad(5,8 \%)$ & $1.613(94,2 \%)$ & 1.713 & $=0,082$ \\
\hline
\end{tabular}


Como se observa na Tabela 3, a prevalência do tabagismo do(a) filho(a) foi de $12,5 \%(99 / 795)$ se apenas o pai fumava, RP $=1,49(12,5 / 8,4)$. Se apenas a mãe fumava, a prevalência foi de $13,4 \%$ (89/663), RP $=1,58$ $(13,4 / 8,5)$. Quando pai e mãe fumavam, a prevalência do tabagismo entre os adolescentes foi de $14,8 \%$ (39/ 263), RP $=1,66(14,8 / 8,9)$ (Tabela 3$)$.

No sexo masculino, $18,4 \%(60 / 326)$ fumavam quando apenas o pai fumava, RP $=1,5(18,4 / 12,7)$ (IC $95 \%=$ $1,09-1,92)$. No feminino, essa prevalência foi de $8,3 \%$ (39/468) (IC $95 \%=6,0-11,2$ ) quando apenas o pai fumava, $\operatorname{RP}=1,6(8,3 / 5,4)($ IC $95 \%=1,07-2,25)$. Entre os adolescentes do sexo masculino, 19,9\% (54/272) faziam uso do cigarro quando apenas a mãe fumava, RP $=1,5$ $(19,9 / 12,9)$ (IC $95 \%=1,15-2,04)$. J á entre os adolescentes do sexo feminino, $9 \%$ (35/390) fumavam quando apenas a mãe fumava, RP $=1,7(9,0 / 5,4)$ (IC $95 \%=1,13$ $1,43)$. Q uando ambos os pais fumavam, 22,1\% (25/ 113) dos adolescentes do sexo masculino fumavam, RP $=1,7$ $(22,1 / 13,4)$ (IC $95 \%=1,13-2,41)$. As meninas apresentaram freqüência de $9,4 \%(14 / 149)$ do uso do cigarro quando ambos os pais fumavam, RP $=1,6(9,4 / 5,8)$ (IC $95 \%=0,94-2,74)$ (Tabela 4).

A média de idade \pm DP da iniciação do tabagismo entre os adolescentes foi de $14,1 \pm 1,7$ anos, com mínimo de 7 e máximo de 19 anos. No sexo masculino essa média foi de 13,9 $\pm 1,7$ anos e no feminino foi de $14,3 \pm 1,6$ anos. 0 início do hábito de fumar aos 10 anos de idade ou menos teve a freqüência de 2,6\% (7/274) (IC 95\% = 1,0-5,2), entre 11 e 13 anos de $32,8 \%$ (90/274) (IC 95\% $=27,3-38)$, entre 14 e 16 anos de 58,0\% (159/274) (IC $95 \%=51,9-63,9)$ e entre 17 e 19 anos de $6,6 \%(18 /$ 274) (IC $95 \%=3,9-10,2$ ), sem diferença estatisticamente significante entre os sexos.

\section{DISCUSSÃO}

0 presente trabalho permitiu estimar a prevalência do tabagismo em uma amostra selecionada de adolescentes escolares e a associação com o tabagismo dos pais nesta amostra. Entretanto, há algumas limitações neste estudo: a constituição da amostra, visto que não foi usado nenhum procedimento aleatório de seleção das escolas, pois se privilegiou a viabilidade da coleta de dados, apesar de a seleção das salas de aula ter sido feita de forma aleatória, para tentar reduzir o viés de seleção de uma amostra por conveniência; os dados colhidos foram obtidos por meio de auto-relato, através de questionário não validado em relação a um padrão, apesar de que a maior parte das suas questões tem validade de conteúdo. ${ }^{(26)}$ Esta limitação foi minimizada utilizando-se como procedimento questionários anônimos, aplicados em sala de aula, em horário letivo, de preenchimento voluntário, havendo garantia verbal, durante a apresentação e a aplicação do questionário, quanto ao caráter confidencial das informações fornecidas. Procedimentos semelhantes foram utilizados em outro estudo no Brasil;(21) a maioria da amostra foi originária de escolas particulares (quatro) e apenas de uma pública, todas localizadas na região metropolitana, fazendo com que os dados possam ser mais aplicados a estudantes da rede particular de ensino de Salvador - Bahia. Isso limita sua generalização à população da rede privada de ensino, da região metropolitana de Salvador, excluindo os não matriculados, os que não estudam e os institucionalizados.

Nas estatísticas americanas observa-se freqüência maior do tabagismo, sem diferença estaticamente significante entre os sexos, exceto na faixa etária de 18 a 21 anos. ${ }^{(29,30)}$ Conforme dados do Centers for Disease Control 19981999, a prevalência do tabagismo foi de $20,6 \%$ entre os matriculados no nível middle school e $34,8 \%$ no high school nos EUA. (31)

Q uando se compara este trabalho com os levantamentos da União Européia (2.000), observa-se que a prevalência do tabagismo entre adolescentes e jovens é maior em alguns países europeus e há diferença entre os sexos, sendo que varia de $13 \%$ a $33 \%$ no sexo feminino e $17 \%$ a $35 \%$ no masculino, conforme o país. Na Grécia, 13\% dos adolescentes do sexo feminino e $22 \%$ do masculino fumavam. ${ }^{(13)}$ Em Portugal, $17 \%$ dos adolescentes do sexo masculino e $22 \%$ dos do sexo feminino fumavam, enquanto que, na França, a freqüência de tabagismo nessa faixa etária foi muito maior que em outros países do continente europeu e em relação ao trabalho atual, atingindo a taxa de $33 \%$ e $35 \%$ entre os sexos feminino e masculino, respectivamente. ${ }^{(13)}$

Diversos levantamentos sobre 0 assunto foram realizados no Brasil com achados semelhantes e diferentes da atual pesquisa: Cotrim et al. (2000), em São Paulo (Brasil), encontraram a prevalência do tabagismo de $13,8 \%$ na rede particular de ensino $(13,6 \%$ no sexo masculino e $15,3 \%$ no feminino) e $5,1 \%(5,6 \%$ no sexo masculino e $4,6 \%$ no feminino) na rede pública. ${ }^{(21)}$ Mauad et al. (1996), em Barretos (SP), verificaram que $7,2 \%$ dos alunos do ensino fundamental fumavam, também sem diferença estatisticamente significante entre os sexos. ${ }^{(32)}$ Muza et al. (1997), em Ribeirão Preto (SP), encontraram a prevalência de $37,7 \%$, com predomínio do sexo masculino. ${ }^{(33)}$ Horta et al. (2001) detectaram prevalência de $11,1 \%$ na zona urbana da cidade de Pelotas (RS). O relatório do Unicef no Brasil (2000/2001) encontrou $12 \%$ de adolescentes fumantes. ${ }^{(34,35)}$ Por outro lado, Carvalho (1987), em Salvador ( $B A)$, detectou $1 \%$ de prevalência do tabagismo entre adolescentes, sem diferença entre os sexos. ${ }^{(19)}$

0 mais recente levantamento no Brasil foi realizado por Carlini (2002), que achou prevalência do tabagismo 
na faixa etária entre 12 e 17 anos de $15,7 \%$, sendo de $15,2 \%$ no sexo masculino e $16,2 \%$ no feminino, aumentando consideravelmente entre 18 e 24 anos para 37,7\%, e havendo diferença entre os sexos. Para a região Nordeste, encontrou $14,3 \%$ de adolescentes fumantes entre 12 e 17 anos, sendo $16,2 \%$ do sexo masculino e $12,6 \%$ do feminino. Também houve aumento considerável na faixa etária de 18 a 24 anos (32,2\%), sendo de 39,9\% no sexo masculino e no feminino, $23,9 \%$. (20) No atual estudo, a amostra de adolescentes escolares apresentou maior prevalência, maior freqüência de experimentação e também maiores taxas de continuidade do hábito após a experimentação.

Neste levantamento, assim como nos trabalhos citados, a prevalência do tabagismo aumentou conforme a idade, porém a diferença entre os sexos variou segundo idade, Estado, região e escolaridade. ${ }^{(20-22,34)} \mathrm{Na}$ presente pesquisa a freqüência foi 2,3 vezes maior entre os rapazes. Este comportamento pode ser confirmado por outros estudos realizados nos Estados Unidos, ${ }^{(36)}$ Europa ${ }^{(13)}$ e América Latina, ${ }^{29,37)}$ havendo aumento da prevalência conforme a idade, porém com variação da diferença entre os sexos. Surpreende que à medida que 0 adolescente cresce, e tem mais noções de comportamento de risco e de prevenção, ${ }^{(38)}$ além de ter consciência dos males do tabaco, ${ }^{(36)}$ passa a fumar com maior freqüência. Isso pode ser explicado pelo fato de que o início e a manutenção do hábito de fumar são multifatoriais, e têm influência da própria característica da idade e sua vulnerabilidade, dos seus pares, dos pais e da conduta deles, e da alta prevalência do tabagismo no meio. $(24,25,36,39-41)$

Entre as raças não foi observada diferença estatisticamente significante na prevalência do hábito de fumar, podendo isso ser justificado pela homogeneidade da amostra neste estudo. Porém, estudos norte-americanos mostram maior prevalência na raça branca. ${ }^{(42)}$

A idade mais freqüente de início do hábito varia de 15 a 16 anos de idade em diversos estudos, podendo chegar a até $8,5 \%$ de freqüência nos adolescentes norte-americanos com idade inferior a 11 anos. ${ }^{(36,37,42-45)}$ Nesse país, em 1992, 10,8\% dos adolescentes haviam fumado seu primeiro cigarro com idade inferior a 10 anos. ${ }^{(29)} \mathrm{A}$ atual pesquisa observou que a idade de iniciação ocorreu mais freqüentemente entre 14 e 16 anos, dados estes coincidentes com os encontrados pelo U nicef (2001) no Brasil $(15 \%)$ e se diferenciando dos de $\mathrm{H}$ orta et al. (2001), que encontraram a maior freqüência de iniciação entre 17 e 18 anos de idade. ${ }^{(34,35)}$

A experimentação do cigarro é o maior fator de risco para o hábito de fumar. (24) Em Salvador (BA), em 1997, a freqüência de experimentação foi de aproximadamente $30 \%$, semelhante à de outros Estados, e menor que nas cidades de Curitiba e Porto A legre, onde atingiu até $45 \%$ de experimentação. ${ }^{(23)} \mathrm{O}$ utro levantamento abordando 0 uso de drogas no Brasil, entre elas o tabaco, não observou diferença estatística entre os sexos na idade de 12 a 17 anos, porém esta diferença ocorreu em idades superiores a 18 anos, em todas as regiões do Brasil. ${ }^{(20)} 0$ presente estudo apontou comportamento semelhante para os adolescentes acima dos 16 anos. Neste estudo foi encontrada maior prevalência do tabagismo entre aqueles que haviam experimentado entre 11 e 13 anos de idade e eram do sexo masculino. Indicando que a experimentação do cigarro ocorreu precocemente e a prevalência do tabagismo tendeu a ser maior nas idades de experimentação inferiores a 13 anos, A dalbjarnardottir \& Rafnsson (2001) observaram maior probabilidade de o adolescente continuar fumando quando a experiência ocorreu aos 14 anos de idade.(24) $\mathrm{O}$ hábito pode durar anos devido ao efeito de tolerância desenvolvido pela nicotina e por sua capacidade de modificar o sistema catecolaminérgico no sistema nervoso central, precocemente e/ ou em longo prazo. ${ }^{(9,46)}$

A influência dos pais é importante na gênese do tabagismo dos adolescentes. ${ }^{(24,25)}$ Carvalho (1987) observou que a maior influência parental no tabagismo dos filhos estava relacionada ao tabagismo da mãe e de ambos os pais em relação à filha, ${ }^{(19)}$ sendo este dado também coincidente com os achados deste estudo, o qual mostrou que, entre filhos de pais e/ ou mães fumantes, havia maior razão de prevalência e, entre as adolescentes, preponderou a influência da mãe. Também neste estudo, quando nenhum dos pais fumava, o adolescente era protegido do hábito. Na U nião Européia, entre 1985 e 1996, o hábito de fumar da mãe aumentava a probabilidade de o adolescente fumar, principalmente os do sexo feminino.(13) Já os achados de Adalbjarnardottir \& Rafnsson (2001), na Islândia, mostraram que essa associação ocorre principalmente aos 14 anos de idade, porém sem diferença entre os sexos. ${ }^{(24)}$ Mesmo havendo outras influências, tais como de amigos, ${ }^{(24)}$ os achados encontrados neste e em outros estudos reforçam a hipótese de que se os pais não fumarem, reduzem o risco de o adolescente fumar.

A pesar de algumas limitações, este trabalho permite tirar várias conclusões em relação ao hábito tabágico em adolescentes escolares na Bahia. A prevalência do hábito de fumar foi de $9,5 \%$ entre os adolescentes, sendo $14 \%$ no sexo masculino e $6 \%$ no feminino. A idade de iniciação do tabagismo foi de $14 \pm 2$ anos. A maioria deles iniciou na idade entre 14 e 16 anos. À medida que aumentou a idade do adolescente, aumentou a prevalência do hábito de fumar. A experimentação do cigarro ocorreu em $46 \%$ dos adolescentes, sendo $53,3 \%$ no sexo masculino e $41 \%$ no feminino. Dos que experimentaram o cigarro, 20,4\% deles continuaram fumando, com maior prevalência no sexo masculino. A prevalência do hábito 
entre adolescentes escolares desta amostra foi maior quando a experimentação ocorreu entre 11 e 13 anos. Nesta amostra houve associação do tabagismo dos pais com 0 dos filhos, sendo maior entre as filhas quando apenas a mãe fumava.

\section{REFERÊNCIAS}

1. Projected smoking-related deaths among youth - U nited States. M MWR M orb Mortal Wkly Rep 1996;45:971-4.

2. Centers for Disease Control and Prevention. Preventing tobacco use among young people. A report of the surgeon general. Executive summary. Recommendations and reports. M MWR Morb Mortal Wkly Rep 1994;43:1-11.

3. Petty TL. Scope of the COPD problem in North America: early studies of prevalence and NHANES III data: basis for early identification and intervention. Chest 2000;117:322S-31S.

4. Galvan Fernandez C, Suarez Lopez de Vergara RG, Oliva Hernandez C, Domenech Martinez E. Patología respiratoria en los jóvenes y hábito tabáquico. Arch Bronconeumol 2000;36:186-90.

5. Nunes de Aguiar VA, Dobashi PD, Novo NF, Bogossian M, Nakatani J, Lopes dos Santos M. Prevalência de sintomas, doenças pulmonares e tabagismo em populações universitárias vivendo em ambientes com níveis diferentes de poluição atmosférica. J Pneumol 1989;15:61-8.

6. Skjoldebrand J, Gahnberg L. Tobacco preventive measures by dental care staff. An attempt to reduce the use of tobacco among adolescents. Swed Dent J 1997;21:49-54.

7. Tomar SL, Winn DM, Swango PA, Giovino GA, Kleinman DV. O ral mucosal smokeless tobacco lesions among adolescents in the United States. J Dent Res 1997;76:1277-86.

8. Eriksen W, Natvig B, Rutle $O$, B ruusgaard D. Smoking and the functional status of young adults. Scand J Prim H ealth Care 1999;17: 174-9.

9. Centers for Disease Control and Prevention. Reasons for tobacco use and symptoms of nicotine withdrawal among adolescent and young adult tobacco users - United States, 1993. MMWR Morb Mortal Wkly Rep 1994;43: 745-50.

10. Centers for Disease Control and Prevention. Current smoking status among adults, 1965-1995. [cited Mar 24]. Available from: http:// www.cdc.gov/ tobacco/ research_data/ adults_prev/ tab_3.htm.

11. Centers for Disease Control and Prevention. Tobacco use among high school students - United States, 1997. MMWR Morb Mortal Wkly Rep 1998;47: 229-33.

12. Centers for Disease Control and Prevention. CDC surveillance summaries, November 18, 1994. MMWR Morb Mortal Wkly Rep 1994; 43:1-44.

13. Sasco AJ, Klehues P. Why can't we convince the young not smoke? Eur J Cancer 1999;35:1933-40.

14. Avedano A, Valenzuela C, Correa F, Almonte C, Sepulveda G. Características del desarrollo psicosocial en adolescentes de 16 a 19 anos en seguimiento longitudinal: I. características de la muestra; consumo de psicotrópicos y hábito de fumar. Rev Chil Pediatr 1988;59:26-33.

15. Ivanovic MD, Castro GC, Ivanovic MR. Factores que inciden en el hábito de fumar de escolares de educación básica y media del Chile. Rev Saude Publica 1997;31:30-43.

16. Daszenies S, Covacevich C. Tabaquismo en estudiantes de enseñanza media de una comunidad rural de Chile. Rev Med Chile 1999;127: 38-43.

17. Ulate F. Fumado en adolescentes. Rev Med Hosp Nac Niños (Costa Rica) 1987;22:59-68.

18. Pinto J, Satander P, Satander J, Weitz P. Consumo de tabaco en populación escolar de Isla de Pascua. Bol Hosp Vina del Mar 1994;50: 185-90.

19. Carvalho FM. Hábito de fumar em adolescentes escolares de Salvador, Bahia. Rev Baiana Saude Publica 1987;14:212-6.

20. Carlini EA, Galduróz J CF, Noto AR, Nappo SA. I Levantamento domiciliar sobre uso de drogas psicotrópicas no Brasil: estudo envolvendo as 107 maiores cidades do país - 2001. São Paulo: CEBRID - Centro de Brasileiro de Informações sobre Drogas Psicotrópicas; UNIFESP - Universidade Federal de São Paulo, SENAD - Secretaria Nacional Antidrogas, Gabinete de Segurança Institucional - Presidência da República; 2002.

21. Cotrim BC, Carvalho CG, Gouveia N. Comportamentos de saúde entre jovens estudantes das redes pública e privada da área metropolitana do Estado de São Paulo. Rev Saude Publica 2000;34:636-45.

22. Muza GM, Bettiol H, Muccillo G, Barbieri MA. Consumo de substâncias psicoativas por adolescentes escolares de Ribeirão Preto, SP (Brasil). I Prevalência do consumo por sexo, idade e tipo de substância. Rev Saude Publica 1997;31:21-9.

23. Goldfarb LCS. Tabagismo: estudos em adolescentes e jovens. In: Schor N, Mota MSFT, Castello Branco VC, editores. Cadernos juventude, saúde e desenvolvimento. Brasília: Ministério da Saúde, Secretaria de Políticas de Saúde, Área de Saúde do Adolescente e do J ovem, 1999; 162-79.

24. Adalbjarnardottir S, Rafnsson FD. Perceived control in adolescent substance use: concurrent and longitudinal analyses. Psychol Addict Behav 2001;15:2532.

25. Graña J L. A nálise dos fatores de risco e de proteção para o consumo de drogas em adolescentes. In: Marinho ML, Caballo VE, editores. Psicologia clínica e da saúde. Londrina: UEL, 2001;55-75.

26. Pereira GM. Epidemiologia, teoria e prática. São Paulo: Guanabara Koogan; 1995.

27. Krieger $\mathrm{H}$, Morton NE, Mi MP, Azevedo E, Freire-Maia A, Yasuda N. Racial admixture in north-eastern Brazil. Ann Hum Genet 1965;29: 113-25.

28. Brasil. Ministério da Educação e Cultura. Censo educacional 2000. Ministério da Educação e Cultura. [citado 25 maio]. Disponível em: http//: www.inep.gov.br/censo/censo2000/censoescolar.asp?MUN= Salvador\&UF $=29 \& B=$ consulta.

29. Adams PF, Schoenborn CA, Moss AJ, Warren CW, Kann L. Health-risk behaviors among our nation's youth: United States, 1992. Vital H ealth Stat $1995 ; 10: 1-51$.

30. U.S. Department of Health and Human Services and Center for Disease Control and Prevention. Youth risk behavior surveillance: United States 1999. Washington: Centers for Disease Control and Prevention, U.S. Department of $\mathrm{H}$ ealth and Human Services, National Center for Chronic Disease Prevention and $\mathrm{H}$ ealth Promotion, $\mathrm{O}$ ffice on Smoking and $\mathrm{H}$ ealth 2000;1-96.

31. Centers for Disease Control and Prevention. Youth tobacco surveillance United States, 1998-1999. Surveillance summaries. MMWR Morb Mortal Wkly Rep 2000;49:1-104.

32. Mauad EC, Bonetti LMG, Silva CM, Nogueira J L, Mirra AP. Prevalência do tabagismo e seus determinantes em algumas escolas de Barretos - São Paulo em 1996. Rev Bras Cancerol 1999;45:41-4.

33. Muza GM, Bettiol H, Muccillo G, Barbieri MA. Consumo de substâncias psicoativas por adolescentes escolares de Ribeirão Preto, SP (Brasil). II Distribuição do consumo por classes sociais. Rev Saude Publica 1997;31: 163-70.

34. Horta BL, Calheiros P, Pinheiro RT, Tomasi E, Costa do Amaral K. Tabagismo em adolescentes de área urbana na região Sul do Brasil. Rev Saude Publica 2001;35:159-64

35. UNICEF. Fundo para Nações U nidas para a Infância. A voz dos adolescentes. Fundo para Nações Unidas para a Infância (UNICEF); 2002.

36. Youth tobacco surveillance - U nited States: 1998-1999. MMWR Morb Mortal Wkly Rep CDC Surveill Summ 2000;49:1-94.

37. Fuentealba F, Cumsille F, A raneda J C, Molina C. Consumo de drogas lícitas e ilícitas en Chile: resultados del estudio de 1998 y comparación con los estudios de 1994 y 1996. Rev Panam Salud Publica 2000;7: 79-87.

38. Boruchovitch E, Mednick BR. Cross-cultural differences in children's concepts of health and illness. Rev Saude Publica 1997;31:448-56.

39. Bayard RT, Bayard J. Socorro! Tengo en hijo adolescente. 20a ed. Buenos Aires: Atlántida; 1997.

40. Luz MTM, Castro e Silva R. Vulnerabilidade e adolescências. In: Nélia S, Tabosa Mota MSF, Castello B ranco V, editores. Cadernos juventude, saúde e desenvolvimento. Brasília: Ministério da Saúde, Secretaria de Políticas de Saúde, Área de Saúde do Adolescente e do J ovem, 1999;93-6. 
41. Gomide PIC. Efeitos das práticas educativas no desenvolvimento do comportamento anti-social. In: Marinho ML, Caballo VE, editores. Psicologia clínica e da saúde. Londrina: UEL, 2001;33-53.

42. U.S. Department of Health and Human Services. Tobacco use among U.S. Racial/ ethnic minority groups - African A mericans, American Indians and Alaska Natives, Asian Americans and Pacific Islanders, and $\mathrm{H}$ ispanics: a report of the surgeon general. Washington: U.S. Department of Health and Human Services, Centers for Disease Control and Prevention, National Center for Chronic Disease Prevention and Health Promotion, Office on Smoking and Health; 1998.
43. Patel DR. Smoking and children. Indian J Pediatr 1999;66:817-24.

44. Pierce JP, Gilpin E. How long will today's new adolescent smoker be addicted to cigarettes? Am J Public Health 1996;86:253-6.

45. Ruiz de Gordejuela E, Lorza JJ , Urresti A. El Tabaquismo entre los trabajadores de Hospital de Santa Marina: consumo y actitudes. Prev Tab 2000;2:95-100.

46. Trauth JA, Seidler FJ, Ali SF, Slotkin TA. Adolescent nicotine exposure produces immediate and long-term changes in CNS noradrenergic and dopaminergic function. Brain Res 2001;892:269-80.

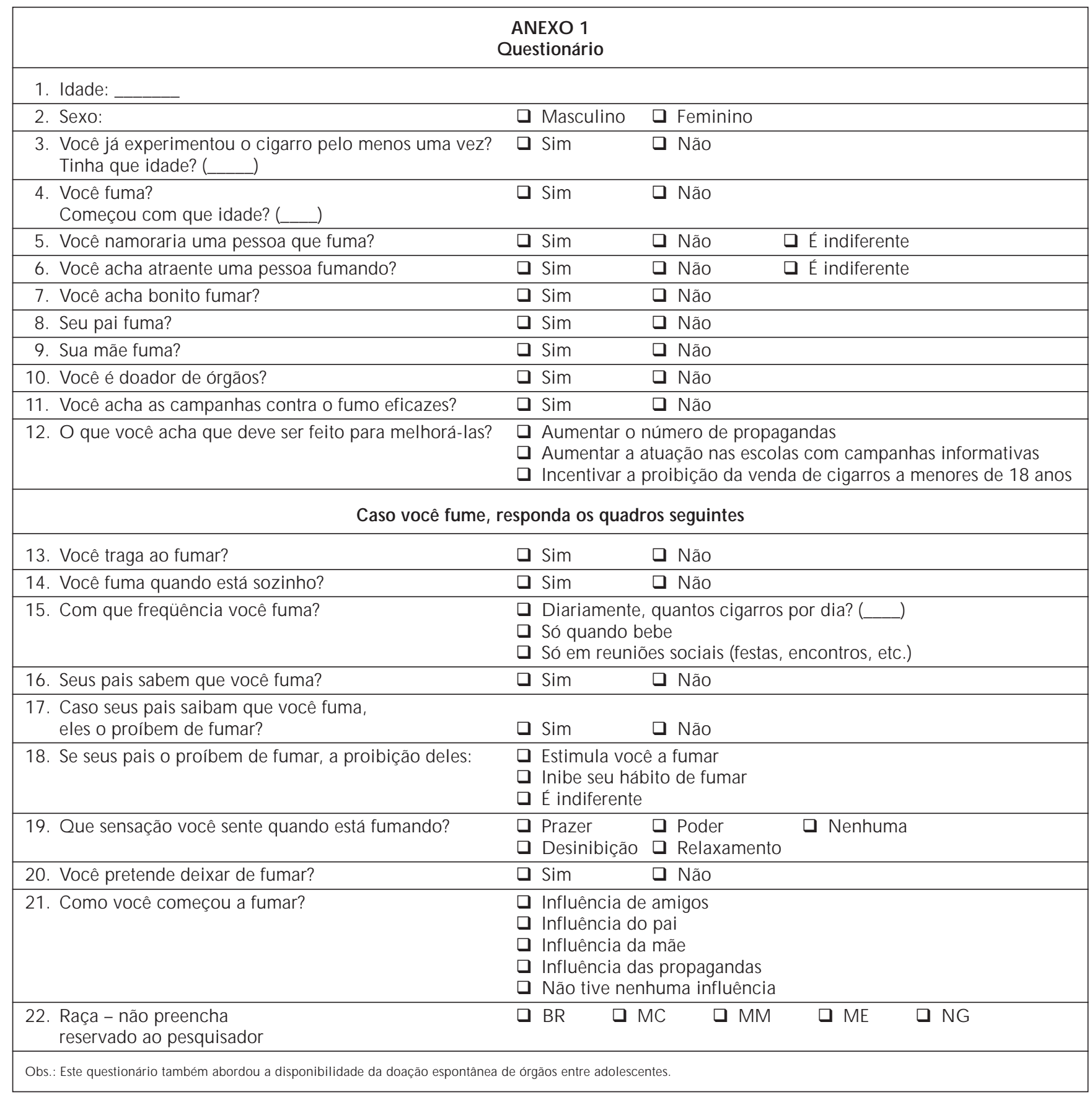

